\title{
Living with and dying from advanced head and neck cancer: a scoping review to assess experiences and unmet needs
}

WHS

Liverpoo nical Commissioning Group
Catriona R Mayland, ${ }^{1,2}$ Stephen Mason, ${ }^{2}$ Hannah C Doughty, ${ }^{2}$ Marilyn Ho, ${ }^{2}$ Prithvi Peddinti, ${ }^{2}$ Praytush Chada, ${ }^{2}$ Simon N Rogers, ${ }^{3,4}$ Cathy Hubbert, ${ }^{5}$ Dominic Macareavy, ${ }^{6}$ Barbara Jack, ${ }^{4}$ Paola Dey ${ }^{4}$

${ }^{1}$ Department of Oncology \& Metabolism, University of Sheffield; ${ }^{2}$ Palliative Care Institute, University of Liverpool; ${ }^{3}$ Aintree University Hospitals NHS Foundation Trust, Liverpool; ${ }^{4}$ Edge Hill University, Ormskirk; ${ }^{5}$ Aintree Park General Practice, Liverpool; Aintree Patient and Carer Head and Neck Research Forum, UK

\section{Aim}

To systematically explore and synthesize current literature on the needs and experiences of patients with advanced head and neck cancer.

\section{Background}

Although studies have examined the integration of specialist palliative care into routine oncological care, none have specifically involved head and neck cancer patients. These patients have especially complex needs due to:

- significant symptom burden

- high level of medical interventions (e.g. feeding tubes)

- risk of acute catastrophic events and fear of how patients will die

psychological concerns affecting patients and families.

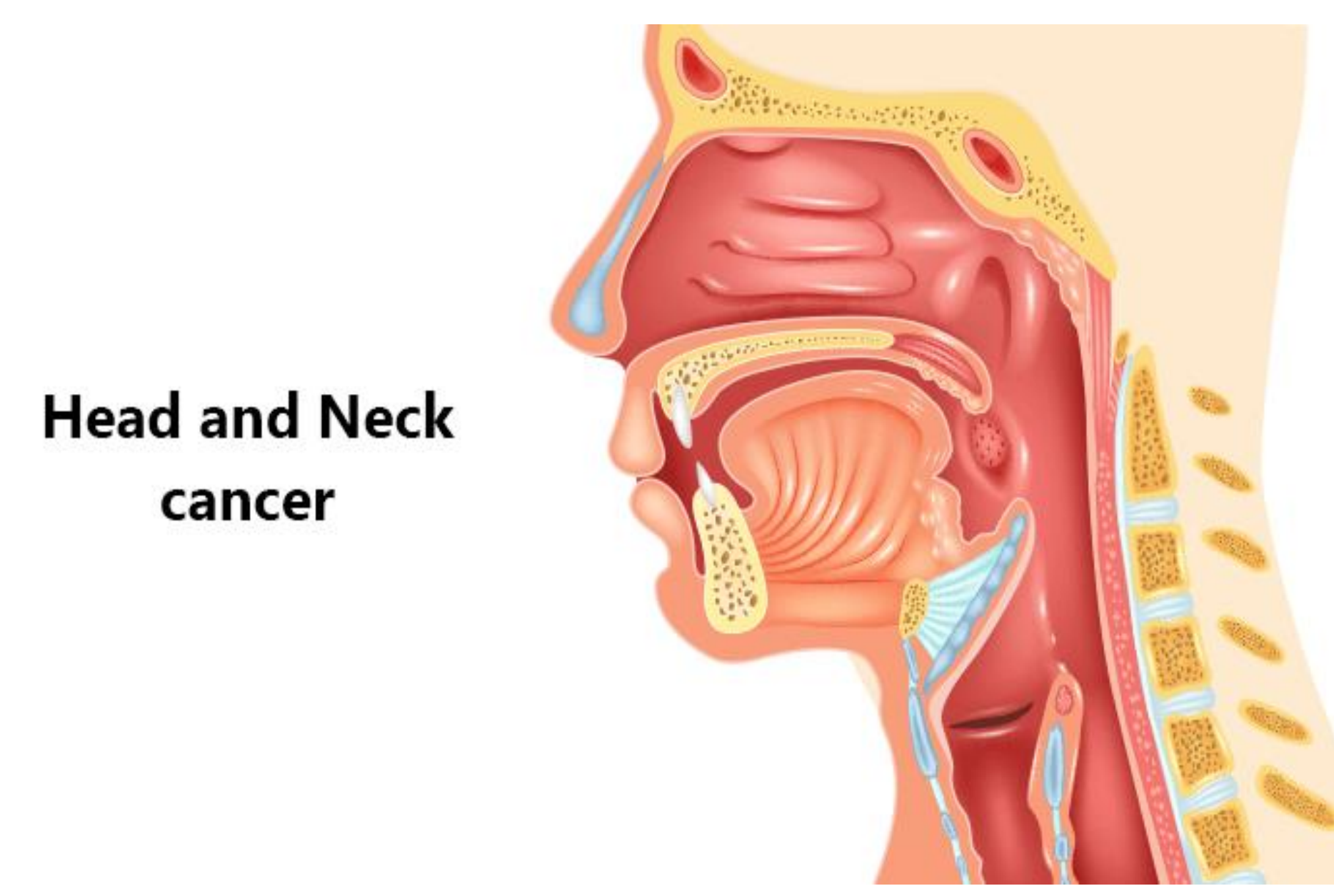

The optimum way to incorporate palliative care within the management of head and neck cancer remains an 'unanswered question' (1). The purpose of this review is to gain a greater understanding of the challenges faced by patients with advanced head and neck cancer, their family carers and the healthcare professionals looking after them.

\section{Methods}

Design: A scoping literature review using systematic methods in keeping with the Joanna Briggs Institute recommendations (2).

\section{Data collection:}

- Electronic databases (MEDLINE (Ovid), EMBASE and CINAHL)

- Searched from January $1996-2017$

Keywords / subject headings:

- 'palliative care' and 'head and neck neoplasms'

Inclusions:

- Empirical studies focused on needs, experiences or views of adults with 'advanced' head and neck

cancer/professionals supporting this population, irrespective of setting or study design;

- Articles published in English.

Exclusions:

- Case reports

Focus on children or survivorship.

Analysis: All abstracts were initially screened by two authors independently (CM and $\mathrm{MH}$ ) to identify potentially eligible papers. The full manuscripts of potentially eligible papers were further screened (by CM, $\mathrm{HD}, \mathrm{PP}$, and $\mathrm{PC}$ ) against eligibility criteria, with a third reviewer (PD or $\mathrm{CM}$ ) resolving any conflicts of opinion, to determine a definitive list of included studies. Data was extracted using a specific proforma (setting, country, population, study design and findings). Findings were tabulated and narratively synthesised into themes.

\section{Results}

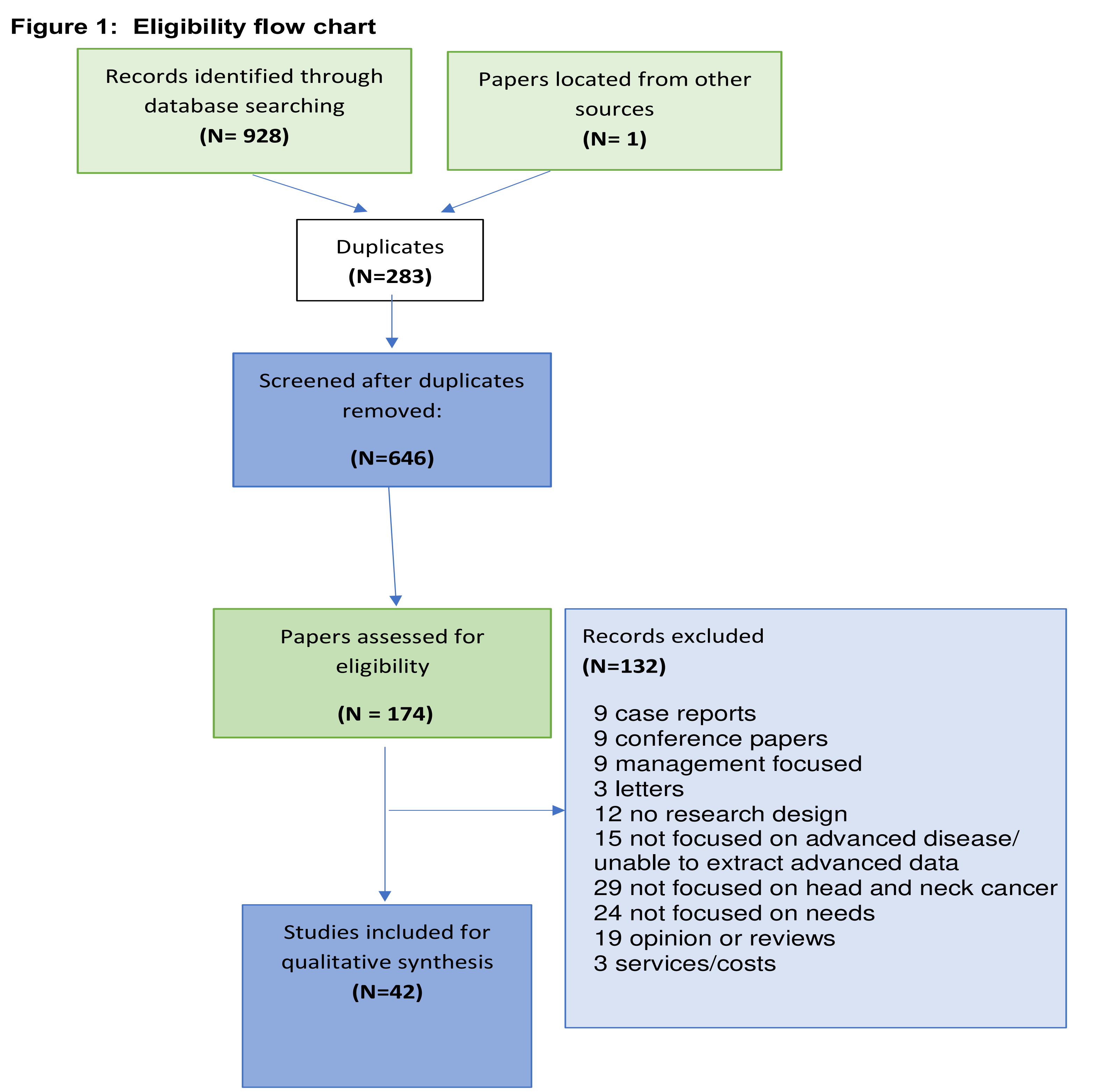

Theme 1: Diverse and multiple symptoms

Average individual has 14 different symptoms = substantial needs (3)

- Including pain (which can be be complex; mixed nociceptive and neuropathic)

- But many others e.g. cough, dysphagia, nausea, malodour, shortness of breath (4)

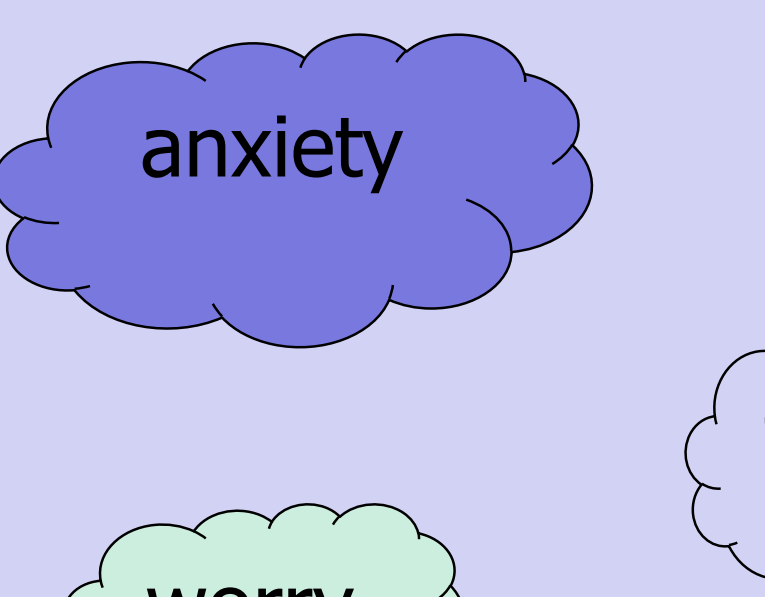

High prevalence of psychological distress $(3,4,5)$ - particular challenges with disfigurement

- High level of anxiety for family carers \& perceived insufficient psychological support

Theme 2: Issues with communicatio

Including

- Patient having communication difficulties (6)

- Differing needs and levels of information between patients and family carers

- Information transfer across care settings sometimes lacking

- High frequency of ethical dilemmas (7)

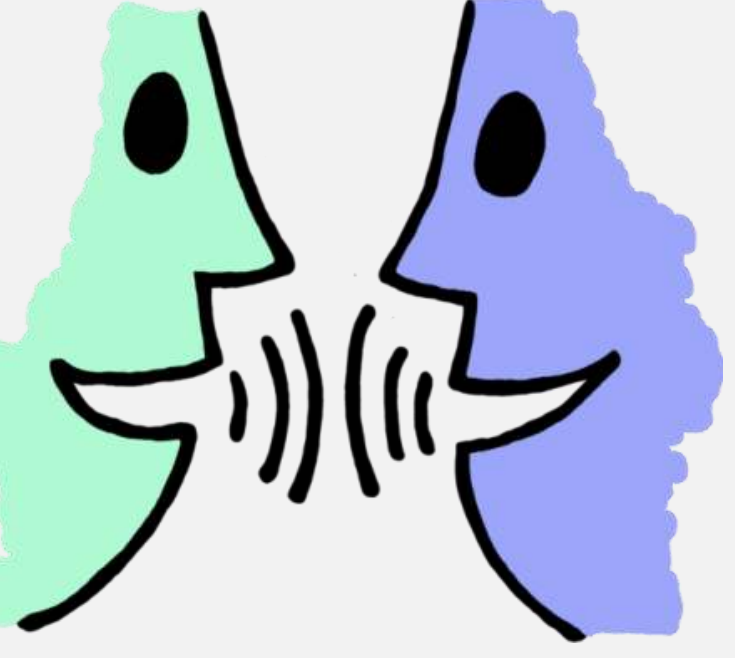

Theme 3: High prevalence of 'intensive interventions' in the last month of life

Including:

- Attendance at 'Accident \& Emergency' or 'Emergency Rooms' (8)

- Admission to hospital / Intensive Care Units

- Cardio-pulmonary resuscitation

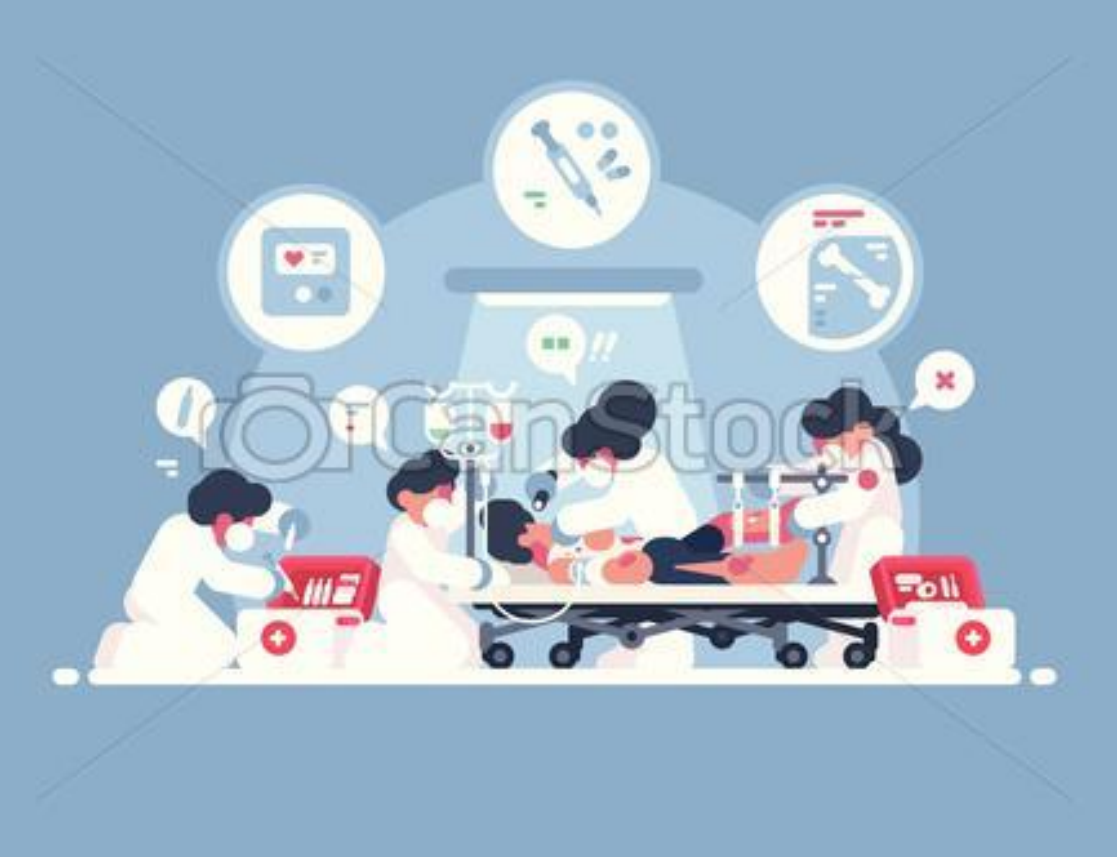

- However, there can be a reluctance for patients to engage with advance care planning (9).

- This seemed linked to an overly optimistic view of aggressive medical intervention while acceptance of palliative care with 'giving up' (9).

\section{Conclusions}

\section{From 928 identified papers, 42 formed the final data set. \\ Main themes: \\ 1. Diverse and multiple symptoms with specific focus on psychological support \\ 2. Issues with communication e.g. differing patient and family needs; transfer of information between healthcare professionals \\ . High prevalence of 'intensive' interventions in the last month of}

